\title{
Gender disparities in the association between socio- demographics and non-communicable disease risk factors among adults with disabilities in Shanghai, China
}

\author{
Youran Zhang ${ }^{1,2}$, Gang Chen ${ }^{\text {Corresp., }}{ }^{1,2}$, Qi Zhang ${ }^{3}$, Jun Lu ${ }^{2}$, Huijiong Yu ${ }^{4}$ \\ 1 Department of Health Law and Health Inspection,School of Public Health, Fudan University, Shanghai, China \\ 2 China Research Center on Disability Issues at Fudan University, Shanghai, China \\ 3 School of Community and Environmental Health, Old Dominion University, Norfolk, Virginia, United States of America \\ 4 Department of Rehabilitation, Shanghai Disabled Persons' Federation, Shanghai, China \\ Corresponding Author: Gang Chen \\ Email address: gchen@shmu.edu.cn
}

Background. Non-communicable disease (NCD) risk factors can co-exist with disability and cause a greater burden on the health status of adults with disabilities. A lack of egalitarian social policies in China may result in gender disparities in the NCD risk factors of adults with disabilities. However, little is known about the gender disparities in the association between socio-demographics and NCD risk factors among adults with disabilities in China; consequently, we examined this association among adults with disabilities in Shanghai, China.

Methods. We used the health examination data of 44,896 adults with disabilities in Shanghai in 2014. Descriptive analyses and logistic regression models were conducted to estimate gender disparities in the association between socio-demographics, disability characteristics, and four selected NCD risk factors among adults with disabilities-including high blood pressure, high blood glucose, high blood lipids, and overweight. We estimated marginal effects (MEs) on NCD risk factors between gender and other confounders.

Results. Women with disabilities were about 11.6 percentage points more likely to suffer from high blood lipids and less likely to develop the other three risk factors than men were. The association of age group, residence permit, education level, marital status, and disability type with health outcomes varied by gender among adults with disabilities. The difference in age effects between men and women was more pronounced in older age groups. Urban residence was associated with less risk of high blood pressure risk among women $(\triangle \mathrm{ME}=-0.035, p<0.01)$, but no significant difference in other NCD risk factors. Education remained a major protective factor against high blood pressure, high blood glucose and overweight among women with disabilities (MEs $<0, p<0.05$ ); however, this did not hold for men. The difference in marriage effects between men and women was observed in high blood lipids ( $\triangle \mathrm{ME}=-$ 0.048 for the married group and -0.054 for the divorced or widowed group) and overweight individuals $(\triangle M E=-0.091$ for the married group and -0.114 for the divorced or widowed group). Women with intellectual disabilities or mental disabilities reported worse health conditions than men did.

Discussion. Preventive strategies and interventions on NCD risk factors for adults with disabilities should take into account gender disparities in these socio-demographic effects. Rural women or poorly educated women with disabilities can be a vulnerable population that requires more health education and promotion strategies. Health education for caregivers of women with intellectual or mental disabilities may also play a vital role in preventing their NCD risk factors. 
1 Gender Disparities in the Association between Socio-Demographics and Non-

2 Communicable Disease Risk Factors among Adults with Disabilities in Shanghai, China

3 Youran Zhang ${ }^{1,2}$, Gang Chen ${ }^{1,2}$, Qi Zhang ${ }^{3}$, Jun Lu ${ }^{2}$, Huijiong Yu ${ }^{4}$

${ }^{1}$ Department of Health Law and Health Inspection, School of Public Health, Fudan University, Shanghai, China

2 China Research Center on Disability Issues at Fudan University, Shanghai, China

3 School of Community and Environmental Health, Old Dominion University, Norfolk, Virginia, USA

${ }^{4}$ Department of Rehabilitation, Shanghai Disabled Persons' Federation, Shanghai, China

Correspondence Author:

Gang Chen ${ }^{1,2}$

1 Department of Health Law and Health Inspection, School of Public Health, Fudan University, Shanghai, China

2 China Research Center on Disability Issues at Fudan University, Shanghai, China

Email address: gchen@shmu.edu.cn

\begin{abstract}
Background. Non-communicable disease (NCD) risk factors can co-exist with disability and cause a greater burden on the health status of adults with disabilities. A lack of egalitarian social policies in China may result in gender disparities in the NCD risk factors of adults with disabilities. However, little is known about the gender disparities in the association between socio-demographics and NCD risk factors among adults with disabilities in China; consequently, we examined this association among adults with disabilities in Shanghai, China.
\end{abstract}

Methods. We used the health examination data of 44,896 adults with disabilities in Shanghai in 2014. Descriptive analyses and logistic regression models were conducted to estimate gender disparities in the association between socio-demographics, disability characteristics, and four selected NCD risk factors among adults with disabilities - including high blood pressure, high blood glucose, high blood lipids, and overweight. We estimated marginal effects (MEs) on NCD risk factors between gender and other confounders.

Results. Women with disabilities were about 11.6 percentage points more likely to suffer from high blood lipids and less likely to develop the other three risk factors than men were. The association of age group, residence permit, education level, marital status, and disability type with health outcomes varied by gender among adults with disabilities. The difference in age effects between men and women was more pronounced in older age groups. Urban residence was associated with less risk of high blood pressure risk among women $(\Delta \mathrm{ME}=-0.035, p<0.01)$, but no significant difference in other NCD risk factors. Education remained a major protective factor against high blood pressure, high blood glucose and overweight among women with disabilities (MEs $<0, p<0.05$ ); however, this did not hold for men. The difference in marriage effects between men and women was observed in high blood lipids ( $\triangle \mathrm{ME}=-0.048$ for the married group and -0.054 for the divorced or widowed group) and overweight individuals $(\triangle \mathrm{ME}$ $=-0.091$ for the married group and -0.114 for the divorced or widowed group). Women with intellectual disabilities or mental disabilities reported worse health conditions than men did.

Discussion. Preventive strategies and interventions on NCD risk factors for adults with disabilities should take into account gender disparities in these socio-demographic effects. Rural 
49

50

51

52

53

54

55

56

57

58

59

60

61

62

63

64

65

66

67

68

69

70

71

72

73

74

75

76

77

78

79

80

81

82

83

84

85

86

87

88

89

90

91

women or poorly educated women with disabilities can be a vulnerable population that requires more health education and promotion strategies. Health education for caregivers of women with intellectual or mental disabilities may also play a vital role in preventing their NCD risk factors.

\section{Introduction}

More than one billion people in the world live with some form of disability, accounting for $15.6 \%$ of the total population [1]. The average prevalence rates of women/men with disabilities are $19.2 \%$ and $12.0 \%$ respectively, ranging from $14.4 \%$ and $9.1 \%$ in developed countries to $22.1 \%$ and $13.8 \%$ in lower income countries [1]. Among them, a population of more than 85 million individuals with disabilities live in China [2-3] and nearly half (48.45\%) are women.

Disabilities, as well as lack of egalitarian social policies and accommodation, severely impact individuals' lives in a variety of ways. Individuals with disabilities are among the most marginalized groups in society, facing high rates of unemployment, poverty and more health challenges [1].

Non-communicable disease (NCD) risk factors, such as uncontrolled high blood pressure, impaired fasting glucose, hyperlipidemia, and overweight, can co-exist with disability and add to the burden on the health status of these populations [4]. These health risks could potentially decrease the balance, strength, endurance, fitness, and flexibility of these individuals and increase the risk of spasticity, depression, and other health problems [5]. When these risk factors further develop into NCD, they can significantly increase mortality and health care expenditure with respect to people with disabilities.

Recent literature has revealed that women have a higher disability rate than men do [6-8]. Although women with disabilities have a longer life expectancy, they tend to suffer more from non-life-threatening conditions. It is well established that there are gender disparities with respect to the risk of developing a disability or the contribution of chronic conditions to the burden of the disability [9-10]. Moreover, some researchers have found that gender differences exist in the prevalence of risk factors among people with disabilities [11-13]. There are also some researchers who have examined the effect of socio-demographic and disability characteristics on NCD risk factors in this population [14-17]. These studies helped build the evidence base for exploring health disparities among them. However, few researchers have sufficiently investigated gender differences in the association between socio-demographics, disability characteristics, and NCD risk factors among people with disabilities, especially in China. To prevent NCD risk factors effectively, Chinese health sectors need to tailor suitable measures for men and women with disabilities respectively. Uncovering how socio-demographic and disability characteristics influence the prevalence of these NCD health risks in men and women with disabilities would be conducive to deploying more accurate interventions.

To examine the gender disparities in a population with disabilities, we used the health examination data of 44,896 adults with disabilities in Shanghai, China. The purpose was to (1) compare population-level estimates of the prevalence of various health risks between men and women with disabilities; and (2) compare the marginal effects (MEs) of socio-demographic and disability characteristics on NCD risk factors between men and women with disabilities.

\section{Materials and Methods}

Data Source and Ethics Statement

Since 2004, Shanghai has provided free yearly health examination services for people with disabilities. Two municipal rehabilitation centers provide professional and comprehensive health 
92 checks for about 40,000 people with disabilities every year. In total 46,108 people with

93 disabilities (accounting for almost $11.49 \%$ of total individuals with disabilities in Shanghai) 94 accessed health examination services at the two municipal rehabilitation centers from January 1, 952014 to December 31, 2014. After eliminating the data of persons aged younger than 18 years (n $96=66)$ or those with missing data on identified variables $(n=1,146), 44,896$ persons with 97 disabilities were included in analyses.

98 The health examination records of these individuals and their socio-demographics were 99 collected by the Shanghai Disabled Persons' Rehabilitation Comprehensive Information Platform 100 (SHDPRCIP), which was established by the Shanghai Disabled Persons' Federation, during this 101 period. The institutional review board (IRB) of the Fudan University School of Public Health 102 (IRB \#2015-08-0563) authorized this study.

103

104

We selected four important NCD risk factors: high blood pressure, high blood glucose, high

\section{Dependent Variables}

Persons with systolic blood pressure $\geq 140 \mathrm{mmHg}$ and/or diastolic pressure $>90 \mathrm{mmHg}$ were denoted as having high blood pressure [18]. A fasting blood glucose level $\geq 6.1 \mathrm{mmol} / \mathrm{L}$ was regarded as high based on Chinese Prevention and Treatment Guideline for Type 2 Diabetes (2013) [19]. Total cholesterol levels $\geq 5.2 \mathrm{mmol} / \mathrm{L}$ or triglyceride levels $\geq 1.7 \mathrm{mmol} / \mathrm{L}$ were classified as high blood lipids according to Chinese Adults' Prevention and Treatment Guidelines for Dyslipidemia (2016) [20]. Moreover, a body mass index $\geq 24 \mathrm{~kg} / \mathrm{m}^{2}$ was considered as overweight based on the guidelines recommended for the Chinese population [21]. All dependent variables were categorized into binary outcomes.

\section{Conceptual Model and Covariates}

The main explanatory variable was gender. Two groups of factors were also controlled: socio-demographics (age group, residence permit, education level, and marital status) and disability characteristics (disability type and disability severity). Residence permit in China contains only two types: urban and rural. Many social security welfare systems, such as health, housing, education and pensions, are based on the household registration system. Urban residence may receive more benefits than rural residence. Age group was defined as 18-29, 30$39,40-49,50-59,60-69$, and 70 or older. The education level groups were elementary school or lower, middle school, high school, and college or higher. Marital status was classified as never married, married, and divorced or widowed.

Based on the Classification and Grading Criteria of Disability (GB/T 26341-2010) [22], specialized medical institutions designated by the Shanghai Disabled Persons' Rehabilitation performed the disability evaluation. The evaluation outcomes (i.e., disability type and disability severity) were registered in the SHDPRCIP. Disability type included hearing disability, speech disability, visual disability, physical disability, intellectual disability, mental disability, and multiple disabilities. Those with hearing disability or speech disability were grouped together [23]. Disability severity was classified into four levels by related function scores of every disability type according to standard Chinese criteria [22]. Level 1 was the most serious disability status and Level 4 was the mildest level. Multiple disabilities refer to two or more 
137 kinds of disabilities. Multiple disabilities are graded according to the grading criteria of the

138

139

140

141

142

143

144

145

146

147

148

149

150

151

152

153

154

155

156

157

158

159

160

161

162

163

164

165

166

167

168

169

170

171

172

173

174

175

176

177

178

179

180

181

182

severest disability.

\section{Statistical Analysis}

Socio-demographics, disability characteristics and NCD risk factors were compared between men and women using standard descriptive methods, such as frequency distribution and chisquare test. Logistic regression models were used to test the associations of the risk factors with socio-demographics and disability factors (model 1: baseline model). Model 2, an interaction model, additionally included the interactions between gender and other covariates. To clearly present the different effects between the two genders, MEs, instead of odds ratios, were estimated in both models, which indicated, on average, that women had more or less probabilities to have these NCD risk factors than men. MEs of socio-demographics and disability factors were estimated separately in men and women to compare the different socio-demographic effect on NCD risk factors between men and women with disabilities.

Four multiple logistic regressions (one for each NCD risk factor) were performed in each model. Stata version 12 was used for all calculations. Statistical significance was considered as $p$ $<0.05$.

\section{Results}

Table 1 presents participants' socio-demographic and disability characteristics according to gender. For the overall sample, the average age $( \pm \mathrm{SD})$ was $56.18 \pm 11.11$ years and $52.98 \%$ were men. Most people were aged 50-59 years (37.60\% men vs. $39.05 \%$ women). About $80 \%$ of the sample were aged older than 50 years. Men had received more education than women had, but were less frequently married. The proportion of men (53.08\%) with physical disabilities was higher than it was for women (46.51\%); however, the proportion of men with visual disabilities was lower (20.85\% in men vs. $26.22 \%$ in women). Compared to women, men were diagnosed as having more severe disabilities. Overall, there were significant differences in the sociodemographic and disability characteristics between men and women, except for residence permit.

The prevalence of NCD risk factors by gender is displayed in Table 2. High blood pressure, high blood lipids, and overweight reached more than $40 \%$ in both gender groups. High blood lipids was the most common of the four selected risk factors in people with disabilities (58.56\%). High blood lipids was most common in women $(64.42 \%)$, which was higher than the rate for men $(53.37 \%)$. However, the proportions of the other three indicators in men were higher than those in women. All the differences were statistically significant.

Figure 1 shows the MEs of women compared with men on NCD risk factors. For example, on average, women were about 11.6 percentage points more likely than men to develop high blood lipids, and women were less likely to develop the other three risk factors. The gender difference in MEs was significant but the difference between two models was non-significant.

Table 3 presents the MEs of demographic and disability characteristics in men and women, respectively, and the gender difference in the MEs of these socio-demographic effects. A positive $\triangle \mathrm{ME}$ means the women's ME is greater than men's ME in values, and vice versa. The gender difference in age effects was more pronounced in older age groups. For example, the $\triangle \mathrm{ME}$ in age effects on high blood pressure was -0.024 and -0.014 for those aged 30-39 and 4049 years, respectively (although non-significant). However, the gender differences in age effects were $0.067,0.073$, and 0.128 in older groups. In other words, age effects in women were smaller than those in men in younger age groups, but significantly larger in older age groups. Similar

PeerJ reviewing PDF | (2017:12:22381:2:0:CHECK 23 Feb 2018) 
183 patterns can be observed for other NCD groups. Urban residence was associated with less risk of 184 high blood pressure among women $(\triangle \mathrm{ME}=-0.035, \mathrm{p}<0.01)$, but no significant difference in 185 other NCD risk factors. In other words, in women, the protective effect of urban residence was 186 much larger and significant than in men with respect to high blood pressure. Education was a 187 protective factor against high blood pressure, high blood glucose and overweight among women 188 with disabilities; i.e. all MEs were negative. More educated women had better health statuses 189 (the absolute values of the MEs increased). However, this did not appear true for men. The $\triangle \mathrm{ME}$ 190 in education was significantly pronounced in these three NCD groups $(\mathrm{p}<0.05)$. Intuitively 191 speaking, education had a significantly protective effect in women, but not in men. The $\triangle \mathrm{ME}$ in 192 marriage effects were observed in high blood lipids $(\Delta \mathrm{ME}=-0.048$ for the married group and 1930.054 for the divorced or widowed group, $p<0.05)$ and overweight $(\Delta \mathrm{ME}=-0.091$ for the 194 married group and -0.114 for the divorced or widowed group, $\mathrm{p}<0.01)$. Regarding disability type effects, gender differences was found in mental disability group and intellectual disability group. The gender difference in the mental disability effect was positive on high blood pressure $(\Delta \mathrm{ME}=0.083, \mathrm{p}<0.01)$, high blood glucose $(\Delta \mathrm{ME}=0.073, \mathrm{p}<0.01)$, and overweight $(\Delta \mathrm{ME}=$ $0.125, \mathrm{p}<0.01)$, but no significant difference in high blood lipids. Women with intellectual disability were more vulnerable to high blood lipids $(\triangle \mathrm{ME}=0.069, \mathrm{p}<0.01)$ and overweight $(\triangle \mathrm{ME}=0.081, \mathrm{p}<0.01)$. Disability severity had a trivial effect on these health risks in both men and women, and the gender differences in disability severity were non-significant.

202

203

204

205

206

207

208

209

210

211

212

213

214

215

216

217

218

219

220

221

222

223

224

225

226

227

\section{Discussion}

In Shanghai, women with disabilities tended to be younger and less educated and were more likely to be married than men were. The prevalence rates of high blood pressure, high blood glucose, and overweight were higher in men with disabilities than in women; however, this was not so for high blood lipids. Our study disclosed the complex gender disparities in sociodemographic effects on NCD risk factors. Previous literature only used gender as simple control variable in the analyses regarding NCD risk factors in adults with disabilities. However, this study suggests the gender effect can vary by different age, education, or disability types.

For example, the changing signs of $\triangle \mathrm{ME}$ across age groups indicated that age generates a strong effect in men on NCD risk factors; however, in older populations, age effects were larger in women, which suggests we may need to pay more attention to chronic illness in elderly women than in elderly men with disabilities [24].

The gender disparity in an urban protective effect is also worth discussion. Since Shanghai has a high degree of urbanization and has a greatly reduced rural-urban gap in health care access [25-26], no significant difference regarding NCD risk factors was observed in men with disabilities. However, rural women can still be a target intervention group to prevent or reduce chronic illness [27-30].

Education remained a major protective factor against these selected risk factors among women with disabilities; although, people with disabilities were less educated than the general population in China [31]. Interestingly, higher education does not play a positive role for men with disabilities. The protective effect was particularly important for women with disabilities. As a result of the segregated labor markets for people with disabilities, women who are more educated may have more opportunities to get jobs, earn more money, and obtain healthier food, and they may also be more likely to take advantage of comprehensive rehabilitation policies and health care utilities [32-33]. Men with disabilities of different educational backgrounds may not 
228

229

230

231

232

233

234

235

236

237

238

239

240

241

242

243

244

245

246

247

248

249

250

251

252

253

254

255

256

257

258

259

260

261

262

263

264

265

266

267

268

269

270

271

272 differ regarding these circumstances above [34]. Poorly educated women with disabilities can be a vulnerable population that needs more health education and promotion strategies.

Results from our analysis showed that marital status was significantly negatively associated with developing high blood pressure and high blood glucose and positively associated with gaining body mass in men. These findings echo the literature demonstrating the association between longevity and better health status with married status, particularly for men [35-36]. However, gender disparities in the association between marriage and the selected health outcomes appeared in high blood lipids and overweight. Although women with disabilities were likely to have health benefits through marriage, they may also suffer from the effects of caregiver burden since they often devote themselves to caring for their family members, especially partners with disabilities. Moreover, being a wife with disabilities could make women feel stressed and find their role restrictive and frustrating, which could lead to unintentional weight loss [37]. All these may explain the less likely to develop high blood lipids and overweight in women than in men.

In general, people with mental disabilities faced worse health outcomes in comparison with those with hearing or speech disabilities. A credible explanation for the high incidence of NCD risk factors in the mental disability group may be less outdoor activity because of their difficulties with communication and social integration. Regarding the gender disparities, women with intellectual disabilities or mental disabilities reported worse health conditions than men did. This is alarming because women may have more sensitive and fragile psychological characteristics, leading to a more closed living environment and worse life state. More aids and supports are needed to help them integrate into the community and adapt to healthy lifestyle [38]. Since women with intellectual disabilities or mental disabilities lack independent decisionmaking ability, including the capacity for health consciousness, health education for their caregivers can play a vital role in preventing their NCD risk factors.

\section{Limitations}

This study had some limitations. First, our study was cross-sectional; therefore, causality cannot be inferred. Since disability and the health risks affect each other over time, it is critical to consider mutual influences to obtain a more accurate representation of gender differences when examining disability. Second, this study did not control for many societal factors, such as the cause of disability, years of disability, employment status, health insurance coverage, individual income, and some behavioral factors, including taking medications for their conditions, physical activity level [39-40]. It is critical for future studies to consider more covariates to determine a more complete picture of gender differences. Finally, this study collected secondary health examination data from the SHDPRCIP. Some people with disabilities, for instance those with severe disabilities or those who received an occupational health examination, may have been unwilling to participate in the initial health examination. Potential selection bias could exist in the sample. Nevertheless, these concerns, to some extent, were mitigated by the large sample size and objective indicators of individuals with disabilities.

\section{Conclusions}

This study highlighted the need for targeted public policies and actions on NCD risk factors prevention and management between men and women with disabilities living in Shanghai. When policymakers target different socio-demographic groups for NCD prevention and intervention, 
273 they need to adopt gender-specific policies to ensure a balanced approach to promote general

274 health in men and women.

275

276

277

278

279

280

281

282

283

284

285

286

287

288

289

290

291

292

293

294

295

296

297

298

299

300

301

302

303

304

305

306

307

308

309

310

311

312

313

314

315

316

\section{Acknowledgments}

We would like to thank Qi Kang, Feixia Liu, Qian Wang, Lian Zong, and Xiaolan Liu for advice.

\section{References}

1. World Health Organization (WHO). World report on disability; WHO: Geneva, Switzerland, 2011.

2. China Disabled Persons' Federation. Communique on Major Statistics of the Second China National Sample Survey on Disability: Leading Group of China National Sample Survey on Disability \& National Bureau of Statistics of the People's Republic of China; China Disabled Persons' Federation: Beijing, China, 2007.

3. The People's Republic of China. The Sixth National Population Census. Available online: http://www.stats.gov.cn/ztjc/zdtjgz/zgrkpc/dlcrkpc/ (accessed on 8 August 2016).

4. World Health Organization (WHO). Global action plan for the prevention and control of noncommunicable diseases 2013-2020; WHO: Geneva, Switzerland, 2013.

5. Rimmer, J.H. Health promotion for people with disabilities: the emerging paradigm shift from disability prevention to prevention of secondary conditions. Phys. Ther. 1999, 79, 495502 .

6. Subramaniam, M.; Abdin, E.; Vaingankar J.A.; Chong, S.A. Gender differences in disability in a multiethnic Asian population: The Singapore Mental Health Study. Compr. Psychiatry 2013, 54, 381-387.

7. Tas, U.; Steyerberg, E.W.; Bierma-Zeinstra, S.M.; Hofman, A.; Koes, B.W.; Verhagen, A.P. Age, gender and disability predict future disability in older people: the Rotterdam Study. BMC Geriatr. 2011, 11, 22.

8. Bora, J.K.; Saikia, N. Gender differentials in self-rated health and self-reported disability among adults in India. PLoS One 2015, 10, e141953

9. Whitson, H.E.; Landerman, L.R.; Newman, A.B.; Fried, L.P.; Pieper, C.F.; Cohen. H.J. Chronic medical conditions and the sex-based disparity in disability: The cardiovascular health study. J. Gerontol. A. Biol. Sci. Med. Sci. 2010, 65A, 1325-1331

10. Yokota, R.T.; de Moura, L.; Andrade, S.S.; de Sa, N.N.; Nusselder, W.J.; Van Oyen, H. Contribution of chronic conditions to gender disparities in disability in the older population in Brazil, 2013. Int. J. Public Health 2016, 61, 1003-1012.

11. Krause, J.S.; Broderick, L. Outcomes after spinal cord injury: Comparisons as a function of gender and race and ethnicity. Arch. Phys. Med. Rehabil. 2004, 85, 355-362.

12. Hinkle, J.L.; Smith, R.; Revere, K. A comparison of stroke risk factors between men and women with disabilities. Rehabil. Nurs. 2006, 31, 70-77.

13. McDermott, S.; Moran, R.; Platt, T.; Dasari, S. Health conditions among women with a disability. J. Womens Health (Larchmt) 2007, 16, 713-720.

14. Chen, G.; Tan, B.K.; Sun, X.; Meng, X.; Jiwa, M. A preliminary report on the medical profile of disabled persons living in Zhabei District, Shanghai, Mainland China. Qual. Prim. Care 2011, 19, 233-244. 
317

318

319

320

321

322

323

324

325

326

327

328

329

330

331

332

333

334

335

336

337

338

339

340

341

342

343

344

345

346

347

348

349

350

351

352

353

354

355

356

357

358

359

360

361

15. Lin, J.D.; Lin, L.P.; Liou, S.W.; Chen, Y.C.; Hsu, S.W.; Liu, C.T. Gender differences in the prevalence of metabolic syndrome and its components among adults with disabilities based on a community health check up data. Res. Dev. Disabil. 2013, 34, 516-520

16. Kang, Q.; Chen, G.; Lu, J.; Yu, H. Health disparities by type of disability: Health examination results of adults (18-64 years) with disabilities in Shanghai, China. PLoS One. 2016, 11 , e155700

17. Horner-Johnson, W.; Dobbertin, K.; Lee, J.C.; Andresen, E.M. Disparities in chronic conditions and health status by type of disability. Disabil. Health J. 2013, 6, 280-6.

18. Revised Commission on Chinese Prevention and Treatment Guideline for Hypertension. Chinese Prevention and Treatment Guideline for Hypertension (2010). Chin. J. Hypertens. 2011, 9, 701-742.

19. Chinese Diabetes Federation. Chinese Prevention and Treatment Guideline for Type 2 Diabetes (2013). Chin. J. Diabete. 2014, 22, 2-42.

20. Revised Joint Commission on Chinese Adults' Prevention and Treatment Guidelines for Dyslipidemia. Chinese Adults' Prevention and Treatment Guidelines for Dyslipidemia (2016). Chinese Circulation Journal. 2016, 31, 937-953.

21. Zhou, B.F. Effect of body mass index on all-cause mortality and incidence of cardiovascular diseases - report for meta-analysis of prospective studies open optimal cut-off points of body mass index in Chinese adults. Biomed. Environ. Sci. 2002, 15, 245-252.

22. Standardization Administration of the People's Republic of China. GB/T 26341-2010 Classification and grading criteria of disability; Beijing, China, 2011.

23. Zheng, X.; Chen, G.; Song, X.; Liu, J.; Yan, L.; Du, W.; Pang, L.; Zhang, L.; Wu, J.; Zhang, B. Zhang, J.; Twenty-year trends in the prevalence of disability in China. Bull. World Health Organ. 2011, 8, 788-797.

24. Hosseinpoor, A.R.; Williams, J.S.; Jann, B.; Kowal, P.; Officer, A.; Posarac, A.; Chatterji, S.; Social determinants of gender differences in disability among older adults: a multi-country decomposition analysis using the World Health Survey. Int. J. Equity Health 2012, 11, 52.

25. Shanghai Municipal Statistics Bureau. Shanghai Statistical Yearbook (2016 Edition); Shanghai Municipal Statistics Bureau: Shanghai, China, 2016

26. Center for Health Statistics and Information. An Analysis Report of National Health Services Survey in China; Center for Health Statistics and Information: Beijing, China, 2013

27. Michele, C.; Franco S.; Jeremy A.; Yong Y.; Veronica G.; Daniel Chisholm. Tackling of unhealthy diets, physical inactivity, and obesity: health effects and cost-effectiveness. Lancet. 2010, 376, 1775-1784.

28. National Health and Family Planning Commission of the People's Republic of China. National Basic Public Health Service Standard (2011 Edition); National Health and Family Planning Commission of the People's Republic of China: Beijing, China, 2011

29. Gaziano, T.A.; Young, C.R.; Fitzmaurice, G.; Atwood, S.; Gaziano, J.M. Laboratory-based versus non-laboratory-based method for assessment of cardiovascular disease risk: The NHANES I Follow-up Study cohort. Lancet. 2008, 371, 923-931.

30. Ibrahim, M.M.; Damasceno, A. Hypertension in developing countries. Lancet. 2012, 380, 611-619.

31. China Disabled Persons' Federation. Monitoring Report on the Status of People with Disabilities and Well-Off Process. China Disabled Persons' Federation: Beijing, China, 2013 
362

363

364

365

366

367

368

369

370

371

372

373

374

375

376

377

378

379

380

381

382

383

32. Piao, H.; He, J.; Zhang, K.; Tang, Z. A cross-sectional study to estimate associations between education level and osteoporosis in a Chinese postmenopausal women sample. Int. J. Clin. Exp. Med. 2015, 8, 21014-21023.

33. Lancet, T. Gender empowerment: beyond education. Lancet. 2011, 378, 1118.

34. Zajacova, A.; Montez, J. Physical functioning trends among US women and men age 45-64 by education level. Biodemography Soc. Biol. 2017, 63, 21-30.

35. August, K.J.; Sorkin, D.H. Sorkin. Marital status and gender differences in managing a chronic illness: The function of health-related social control. Soc. Sci. Med. 2010, 71, 18311838

36. Wilson, S.E. Marriage, gender and obesity in later life. Econ. Hum. Biol. 2012, 10, 431-453

37. Trevisan, C.; Veronese, N.; Maggi, S.; Baggio, G.; De, Rui, M.; Bolzetta, F.; Zambon, S.; Sartori, L.; Perissinotto, E.; Crepaldi, G.; Manzato, E.; Sergi, G.; Marital status and frailty in older people: Gender differences in the Progetto Veneto Anziani Longitudinal Study. $J$. Women's Health 2016 25, 630-637.

38. Merrick, J.; Kandel, I.; Morad, M. Health needs of adults with intellectual disability relevant for the family physician. Sci. World J. 2003, 3, 937-945

39. Sahlin, K.B.; Lexell, J.; Impact of organized sports on activity, participation, and quality of life in people with neurologic disabilities. PM R. 2015, 7, 1081-1088.

40. Carroll, D.D.; Courtney-Long, E.A.; Stevens, A.C.; Sloan, M.L.; Lullo, C.; Visser, S.N.; Fox, M.H.; Armour, B.S.; Campbell, V.A.; Brown, D.R.; Dorn, J.M.; Vital signs: disability and physical activity--United States, 2009-2012. MMWR, Morb. Mortal. Wkly. Rep. 2014, $63,407-413$. 


\section{Table $\mathbf{1}$ (on next page)}

Socio-demographic and disability characteristics of the sample according to gender. 
Table 1. Socio-demographic and disability characteristics of the sample according to gender

\begin{tabular}{|c|c|c|c|c|c|c|c|}
\hline \multirow[t]{2}{*}{ Characteristics } & \multicolumn{2}{|c|}{ Total } & \multicolumn{2}{|c|}{ Men } & \multicolumn{2}{|c|}{ Women } & \multirow[t]{2}{*}{$p$} \\
\hline & n & $\%$ & n & $\%$ & $\mathbf{n}$ & $\%$ & \\
\hline \multicolumn{8}{|l|}{ Age group } \\
\hline $18-29$ & 1406 & 3.13 & 768 & 3.23 & 638 & 3.02 & $<0.01$ \\
\hline $30-39$ & 2740 & 6.10 & 1376 & 5.79 & 1364 & 6.46 & \\
\hline $40-49$ & 5121 & 11.41 & 2483 & 10.44 & 2638 & 12.50 & \\
\hline $50-59$ & 17185 & 38.28 & 8942 & 37.60 & 8243 & 39.05 & \\
\hline $60-69$ & 14875 & 33.13 & 8184 & 34.41 & 6691 & 31.69 & \\
\hline $70+$ & 3569 & 7.95 & 2032 & 8.54 & 1537 & 7.28 & \\
\hline \multicolumn{8}{|l|}{ Residence permit } \\
\hline Rural & 9271 & 20.65 & 4883 & 20.53 & 4388 & 20.79 & 0.50 \\
\hline Urban & 35,625 & 79.35 & 18902 & 79.47 & 16723 & 79.21 & \\
\hline \multicolumn{8}{|l|}{ Education level } \\
\hline Elementary school or lower & 10,737 & 23.92 & 4950 & 20.81 & 5787 & 27.41 & $<0.01$ \\
\hline Middle school & 22,179 & 49.40 & 12,083 & 50.80 & 10,096 & 47.82 & \\
\hline High school & 9985 & 22.24 & 5423 & 22.80 & 4562 & 21.61 & \\
\hline College or higher & 1995 & 4.44 & 1329 & 5.59 & 666 & 3.15 & \\
\hline \multicolumn{8}{|l|}{ Marital status } \\
\hline Never married & 5834 & 12.99 & 4021 & 16.91 & 1813 & 8.59 & $<0.01$ \\
\hline Married & 36,137 & 80.49 & 18,430 & 77.49 & 17,707 & 83.88 & \\
\hline Divorced or widowed & 2925 & 6.52 & 1334 & 5.61 & 1591 & 7.54 & \\
\hline \multicolumn{8}{|l|}{ Disability type } \\
\hline Hearing or speech & 4417 & 9.84 & 2320 & 9.75 & 2097 & 9.93 & $<0.01$ \\
\hline Visual & 10,496 & 23.38 & 4960 & 20.85 & 5536 & 26.22 & \\
\hline Physical & 22,443 & 49.99 & 12,625 & 53.08 & 9818 & 46.51 & \\
\hline Intellectual & 5013 & 11.17 & 2639 & 11.10 & 2374 & 11.25 & \\
\hline Mental & 2019 & 4.50 & 951 & 4.00 & 1068 & 5.06 & \\
\hline Multiple & 508 & 1.13 & 290 & 1.22 & 218 & 1.03 & \\
\hline \multicolumn{8}{|l|}{ Disability severity } \\
\hline Level 1 & 3583 & 7.98 & 1943 & 8.17 & 1640 & 7.77 & $<0.01$ \\
\hline Level 2 & 5865 & 13.06 & 3283 & 13.80 & 2582 & 12.23 & \\
\hline Level 3 & 13,027 & 29.02 & 7362 & 30.95 & 5665 & 26.83 & \\
\hline Level 4 & 22,421 & 49.94 & 11197 & 47.08 & 11,224 & 53.17 & \\
\hline
\end{tabular}


Table 2 (on next page)

NCD risk factors according to gender. 
Table 2. NCD risk factors according to gender.

\begin{tabular}{|c|c|c|c|c|c|c|c|}
\hline & \multicolumn{2}{|c|}{ Total } & \multicolumn{2}{|c|}{ Men } & \multicolumn{2}{|c|}{ Women } & \multirow[t]{2}{*}{$p$} \\
\hline & $\mathrm{n}$ & $\%$ & $\mathrm{n}$ & $\%$ & $\mathrm{n}$ & $\%$ & \\
\hline High blood pressure & 19180 & 42.72 & 10548 & 44.35 & 8632 & 40.89 & $<0.01$ \\
\hline High blood glucose & 8869 & 19.75 & 5114 & 21.50 & 3755 & 17.79 & $<0.01$ \\
\hline High blood lipids & 26293 & 58.56 & 12693 & 53.37 & 13600 & 64.42 & $<0.01$ \\
\hline Overweight & 22091 & 49.20 & 12176 & 51.19 & 9915 & 46.97 & $<0.01$ \\
\hline
\end{tabular}




\section{Table 3 (on next page)}

MEs of demographic and disability characteristics between men and women with disabilities in interaction model. 
Table 3. MEs of demographic and disability characteristics between men and women with disabilities in interaction model

\begin{tabular}{|c|c|c|c|c|c|c|c|c|c|c|c|c|}
\hline & \multicolumn{3}{|c|}{ High blood pressure } & \multicolumn{3}{|c|}{ High blood glucose } & \multicolumn{3}{|c|}{ High blood lipids } & \multicolumn{3}{|c|}{ Overweight } \\
\hline & Men & Women & $\Delta \mathbf{M E}$ & Men & Women & $\Delta \mathbf{M E}$ & Men & Women & $\Delta \mathbf{M E}$ & Men & Women & $\Delta \mathbf{M E}$ \\
\hline \multicolumn{13}{|l|}{ Age group } \\
\hline $18-29$ & Reference & & & & & & & & & & & \\
\hline $30-39$ & $0.059 * *$ & $0.035^{*}$ & -0.024 & $0.061 * *$ & $0.029 * *$ & $-0.031 *$ & $0.088^{* *}$ & $0.047 *$ & -0.041 & 0.014 & -0.029 & -0.043 \\
\hline $40-49$ & $0.132 * *$ & $0.119 * *$ & -0.014 & $0.115^{* *}$ & $0.068 * *$ & $-0.048 * *$ & $0.149 * *$ & $0.155^{* *}$ & 0.006 & -0.007 & 0.006 & 0.013 \\
\hline $50-59$ & $0.216^{* *}$ & $0.283^{* *}$ & $0.067 * *$ & $0.180 * *$ & $0.137 * *$ & $-0.044 * *$ & $0.142 * *$ & $0.369 * *$ & $0.226^{* *}$ & $-0.051^{*}$ & 0.043 & $0.094^{* *}$ \\
\hline $60-69$ & $0.312 * *$ & $0.385^{* *}$ & $0.073 * *$ & $0.197 * *$ & $0.163^{* *}$ & $-0.035^{*}$ & $0.106^{* *}$ & $0.418^{* *}$ & $0.312 * *$ & -0.037 & $0.081 * *$ & $0.118^{* *}$ \\
\hline $70+$ & $0.420 * *$ & $0.548 * *$ & $0.128 * *$ & $0.214 * *$ & $0.204 * *$ & -0.009 & 0.029 & $0.406^{* *}$ & $0.377 * *$ & $-0.052 *$ & $0.126^{* *}$ & $0.178^{* *}$ \\
\hline \multicolumn{13}{|l|}{ Residence permit } \\
\hline rural & Reference & & & & & & & & & & & \\
\hline urban & -0.008 & $-0.043 * *$ & $-0.035 * *$ & 0.012 & -0.001 & -0.013 & 0.013 & $0.021^{*}$ & 0.008 & $0.036 * *$ & $0.023 * *$ & -0.013 \\
\hline \multicolumn{13}{|l|}{ Education level } \\
\hline \multicolumn{13}{|c|}{ elementary school or lower Reference } \\
\hline middle school & 0.011 & $-0.032 * *$ & $-0.043 * *$ & 0.005 & $-0.022 * *$ & $-0.027 * *$ & 0.015 & 0.004 & -0.011 & 0.000 & $-0.069 * *$ & $-0.068 * *$ \\
\hline high school & $0.025^{*}$ & $-0.067 * *$ & $-0.092 * *$ & 0.007 & $-0.039 * *$ & $-0.045^{* *}$ & $0.024 *$ & $0.027 * *$ & 0.003 & 0.008 & $-0.115 * *$ & $-0.122 * *$ \\
\hline college or higher & 0.009 & $-0.097 * *$ & $-0.106 * *$ & $-0.036 * *$ & $-0.066^{* *}$ & -0.030 & 0.030 & 0.029 & -0.001 & 0.014 & $-0.140 * *$ & $-0.154 * *$ \\
\hline \multicolumn{13}{|l|}{ Marital status } \\
\hline never married & Reference & & & & & & & & & & & \\
\hline married & $-0.031 * *$ & -0.029 & 0.002 & $-0.020^{*}$ & 0.000 & 0.020 & 0.014 & $-0.034 *$ & $-0.048 * *$ & $0.092 * *$ & 0.001 & $-0.091 * *$ \\
\hline divorced or widowed & $-0.033^{*}$ & -0.019 & 0.014 & -0.024 & -0.006 & 0.018 & 0.016 & $-0.038^{*}$ & $-0.054^{*}$ & $0.081 * *$ & -0.032 & $-0.114 * *$ \\
\hline
\end{tabular}

Disability type 


\begin{tabular}{lllllllllllll} 
hearing or speech & Reference & & & & & & & & & \\
visual & 0.010 & 0.010 & 0.000 & $0.030^{* *}$ & 0.004 & -0.026 & 0.013 & 0.018 & 0.005 & 0.013 & 0.022 & 0.008 \\
physical & 0.019 & 0.000 & -0.019 & 0.013 & -0.009 & -0.022 & 0.019 & 0.017 & -0.002 & $0.045^{* *}$ & $0.065^{* *}$ & 0.021 \\
intellectual & 0.005 & 0.017 & 0.012 & 0.013 & $0.045^{* *}$ & 0.032 & $-0.059^{* *}$ & 0.010 & $0.069^{* *}$ & 0.023 & $0.104^{* *}$ & $0.081^{* *}$ \\
mental & $-0.092^{* *}$ & -0.009 & $0.083^{* *}$ & 0.025 & $0.098^{* *}$ & $0.073^{* *}$ & $0.046^{*}$ & $0.044^{*}$ & -0.003 & $0.108^{* *}$ & $0.233^{* *}$ & $0.125^{* *}$ \\
multiple & 0.008 & $-0.078^{*}$ & -0.086 & -0.018 & 0.019 & 0.037 & 0.023 & 0.043 & 0.019 & -0.004 & -0.013 & -0.009 \\
Disability severity & & & & & & & & & & & & \\
level 1 & Reference & & & & & & & & & & \\
level 2 & $0.036^{*}$ & -0.019 & $-0.055^{* *}$ & 0.004 & 0.007 & 0.004 & -0.026 & -0.021 & 0.005 & 0.009 & -0.005 & -0.014 \\
level 3 & -0.016 & 0.009 & 0.024 & $-0.027^{*}$ & -0.014 & 0.013 & -0.021 & -0.026 & -0.004 & -0.015 & 0.003 & 0.017 \\
level 4 & 0.008 & 0.019 & 0.011 & -0.005 & 0.005 & 0.010 & $-0.028^{*}$ & -0.024 & 0.004 & 0.003 & 0.010 & 0.007 \\
\hline
\end{tabular}

$* p<0.05$ with logistic regression; $* * p<0.01$ with logistic regression. 
Figure 1

\section{MEs of Women vs. Men on NCD Risk Factors.}

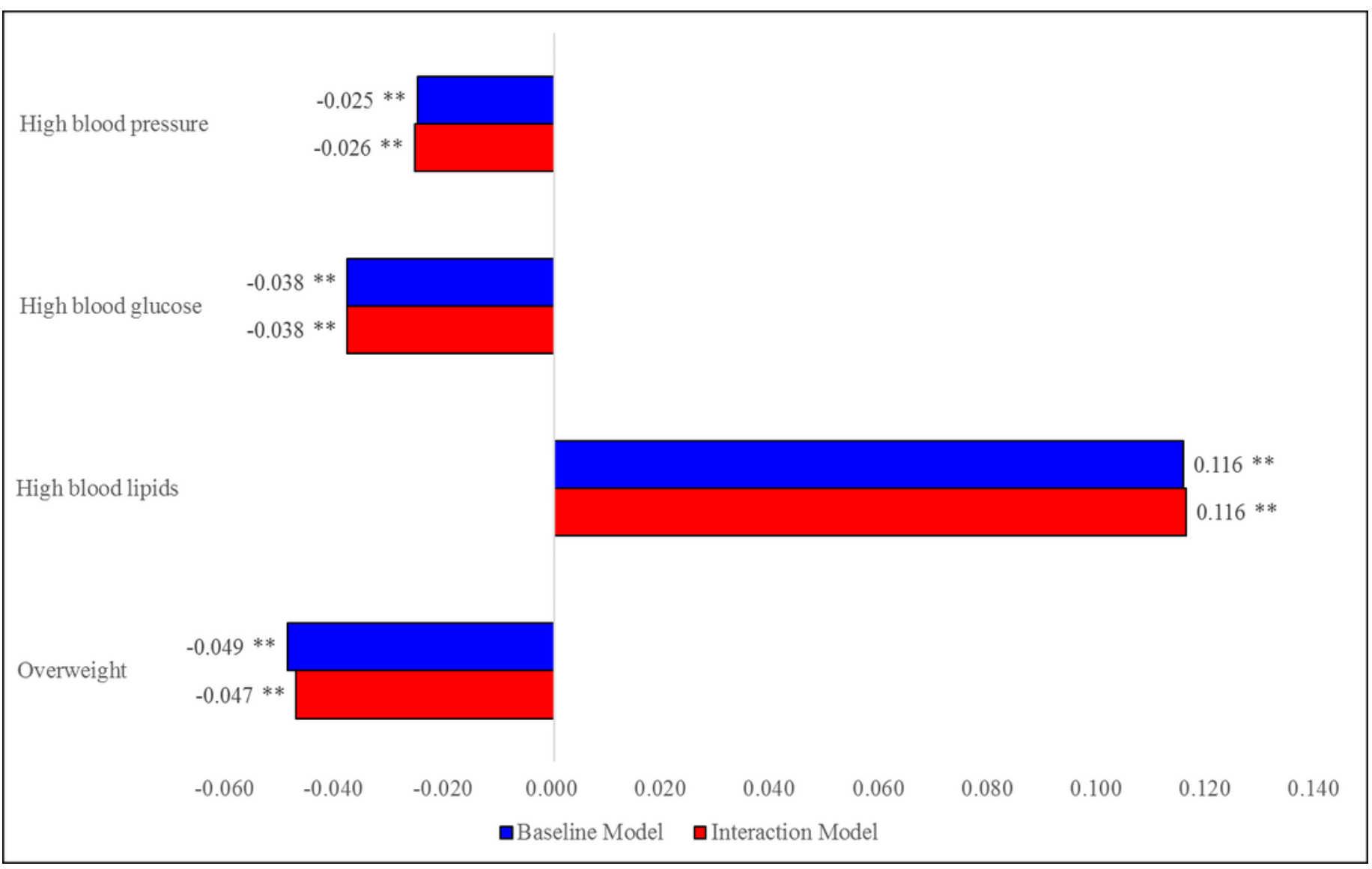

